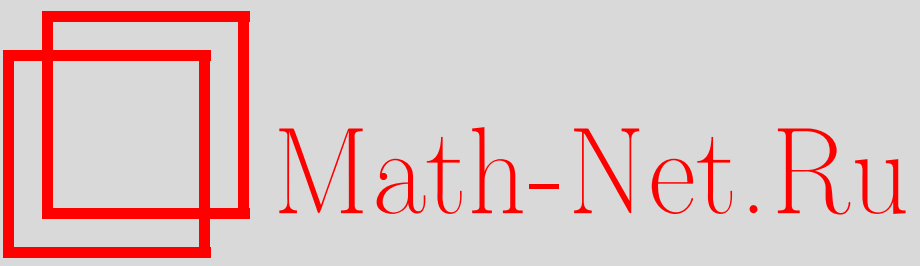

В. В. Пупышев, Ложные решения уравнений Фаддеева с центральными потенциалами, ТМФ, 1996, том 107, номер 3, 501-512

DOI: https://doi.org/10.4213/tmf1171

Использование Общероссийского математического портала Math-Net.Ru подразумевает, что вы прочитали и согласны с пользовательским соглашением

http://www . mathnet.ru/rus/agreement

Параметры загрузки:

IP: 54.174 .149 .18

26 апреля 2023 г., 12:42:53 


\section{ЛОЖНЫЕ РЕШЕНИЯ УРАВНЕНИЙ ФАДДЕЕВА С ЦЕНТРАЛЬНЫМИ ПОТЕНЦИАЛАМИ}

В рамках метода гипергармоник исследуются ложные решения дифференциальных уравнений Фаддеева для системы трех различных частиц, взаимодействующих посредством центральных потенциалов. Доказывается критерий существования ложных решений. Предлагается простой способ построения ложных решений в явном виде. Полученные результаты поясняются примерами.

\section{1. ВВЕДЕНИЕ}

Математически корректная формулировка квантовой задачи трех частиц, взаимодействуюших посредством парных потенциалов $V_{i}$, впервые была дана Л.Д. Фаддеевым в импульсном пространстве [1].

Позднее наряду с интегральными уравнениями Фаддеева для компонент $T$-матрицы стали интенсивно применяться дифференциальные уравнения Фаддеева

$$
\left(H_{0}-E\right) \Psi_{i}=-V_{i} \Psi=-V_{i} \sum_{k=1}^{3} \Psi_{k}
$$

для трех $(i=1,2,3)$ компонент $\Psi_{i}$ волновой функции

$$
\Psi=\sum_{k=1}^{3} \Psi_{k}
$$

в шестимерном координатном пространстве $\mathcal{R}^{6}$. Главная проблема квантовой теории трех частиц в координатном пространстве заключалась в выводе физических граничных условий, гарантируюших существование и единственность решений уравнений (1). Основной вклад в решение этой сложной задачи был сделан С.П. Меркурьевым в его собственных работах и работах, выполненных в соавторстве. Ссылки на них даны в книге [2] и обзорах [3-5].

Настояшая работа посвяшена специальным решениям уравнений (1). В этом разделе мы поясним, почему такие решения могут сушествовать и почему их исследование представляется интересным. 
В уравнении Шредингера

$$
\left(H_{0}-E\right) \Psi=\left(-\sum_{k=1}^{3} V_{k}\right) \Psi
$$

неизвестной функцией $\Psi$ является вся фаддеевская сумма (2). В системе (1) уравнений Фаддеева неизвестными функциями являются слагаемые этой суммы. Вообше говоря, сумма трех некоторых функций, отличных от нуля, может быть тождественно равной нулю. Поэтому система (1), не дополненная какими-либо граничными условиями, может иметь нетривиальное решение $\left\{\Psi_{1}, \Psi_{2}, \Psi_{3}\right\}$, сумма (2) компонент которого тождественно равна нулю, $\Psi \equiv 0$. Такое решение (обозначим его совокупностью $\left\{S_{1}, S_{2}, S_{3}\right\}$ ) обращает в нуль одновременно как правую, так и левую части системы (1):

$$
\begin{aligned}
\sum_{k=1}^{3} S_{k} & =0, \\
\left(H_{0}-E\right) S_{i} & =0 .
\end{aligned}
$$

Совокупность $\left\{S_{1}, S_{2}, S_{3}\right\}$ дважды непрерывно дифференцируемых функций, удовлетворяюших только системе уравнений (4), (5), принято [6] называть ложным решением уравнений (1). Так как ложное решение удовлетворяет свободному уравнению Шредингера (5), то оно не содержит никакой информации о парных потенциалах. Согласно (4) ложное решение отвечает тривиальному решению $(\Psi \equiv 0)$ уравнения Шредингера (3). Следовательно, ложное решение не соответствует никакому физическому состоянию системы трех частиц.

Тем не менее ложным решениям было посвяшено много работ. Факт сушествования ложных решений отмечался многими авторами (см. обзоры $[6,7]$ и оригинальные работы [8-15]). Обшие свойства трехчастичного гамильтониана, порождаюшие ложные решения, и такие общие вопросы, как полнота пространства ложных и физических решений, исследовались в работах $[8,9]$ и в последуюшей работе [10].

Точные решения уравнений (1) известны лишь в случае осцилляторных потенциалов [11] и потенциалов, обратно пропорциональных квадрату расстояния [13]. Уравнения (4) и (5) не содержат потенциалов, что сушественно упрошает построение их точных решений. Ложные решения, найденные аналитически, можно использовать как эталонные для тестирования алгоритмов численного решения трехчастичных уравнений (1) и для решения четырехчастичных задач методом разложения по трехчастичному биортогональному базису, который ложные решения образуют в совокупности с физическими трехчастичными решениями [9].

Однако в явном виде ложные решения найдены лишь в частных случаях, а именно для системы трех тож дественных частиц, взаимодействуюших посредством $S$-волновых парных потенциалов и находящихся в состоянии с полным угловым моментом, равным нулю $[11,12,15]$ или единице $[13,14]$. Следует также отметить, что в общем случае, т.е. для произвольной трехчастичной системы, критерий сушествования ложных решений и простой способ построения таких решений в явном виде до сих пор неизвестны. 
В настоящей работе мы исследуем задачу трех частиц с центральными парными взаимодействиями. В разделе 2 мы опишем обозначения и приведем формулы, известные в теории гипергармоник [16-20]. Используя эти формулы и теорию матриц [21], в разделе 3 мы докажем критерий сушествования ложных решений и покажем, как их можно построить в явном виде. Полученные результаты мы поясним простыми примерами в разделе 4 и сравним с известными в разделе 5 .

\section{2. ОБОЗНАЧЕНИЯ И ОСНОВНЫЕ ФОРМУЛЫ}

Пусть три бесспиновые частицы имеют различные массы $m_{1}, m_{2}$ и $m_{3}$, а потенциалы $V_{i}$, действуюшие в каждой из трех $(i=1,2,3)$ пар частищ, обладают сферической симметрией: $V_{i}\left(\vec{x}_{i}\right)=V_{i}\left(x_{i}\right)$.

Квантовые состояния рассматриваемой системы определяются совокупностью $\varepsilon \equiv$ $\{l, m, \sigma\}$ трех квантовых чисел: полного углового момента $l$, его проекции на ось $O Z$ некоторой фиксированной в шестимерном координатном пространстве $\mathcal{R}^{6}$ системы координат и четности $\sigma= \pm 1$ относительно инверсии $\left(\vec{x}_{i}, \vec{y}_{i}\right) \rightarrow\left(-\vec{x}_{i},-\vec{y}_{i}\right)$ любой пары приведенных векторов Якоби. Различные пары $(k \neq i)$ таких векторов связаны унитарным преобразованием [16]

$$
\left(\begin{array}{l}
\vec{x}_{k} \\
\vec{y}_{k}
\end{array}\right)=-\cos \gamma_{k, i}\left(\begin{array}{cc}
1 & \operatorname{tg} \gamma_{k, i} \\
-\operatorname{tg} \gamma_{k, i} & 1
\end{array}\right)\left(\begin{array}{l}
\vec{x}_{i} \\
\vec{y}_{i}
\end{array}\right)
$$

Кинематический угол преобразования (6),

$$
\gamma_{k, i} \equiv(-1)^{p} \operatorname{arctg}\left(\frac{m_{j}\left(m_{1}+m_{2}+m_{3}\right)}{m_{i} m_{k}}\right)^{1 / 2}
$$

зависит лиш от масс частиц и числа $p$. Оно четное или нечетное в зависимости от четности перестановки триады $(1,2,3)$, дающей последовательность $(k, j, i)$.

Каждой паре $\left(\vec{x}_{i}, \vec{y}_{i}\right)$ соответствует стандартный набор $\left(r, \Omega_{i}\right)$ гиперсферических координат [16]: $r \equiv\left(x_{i}^{2}+y_{i}^{2}\right)^{1 / 2}, \Omega_{i} \equiv\left\{\hat{x}_{i}, \hat{y}_{i}, \varphi_{i}\right\}$, где $\varphi_{i} \equiv \operatorname{arctg}\left(y_{i} / x_{i}\right) \subset[0, \pi / 2]$, а $\hat{c}-$ совокупность двух сферических углов вектора $\vec{c}$.

Ложные решения мы будем искать в линейном пространстве $\mathcal{A}^{\varepsilon}$ дважды непрерывно дифференцируемых по всем своим шести аргументам функций, обладающих набором $\varepsilon$ квантовых чисел $l, m, \sigma$.

Бисферические гармоники [18], обладающие квантовыми числами $\left\{l_{x_{i}}, l_{y_{i}}, l, m, \sigma\right\}$, образуют в пространстве $\mathcal{A}^{\varepsilon}$ ортонормированный базис на торе $\mathcal{T}^{4} \equiv \mathcal{S}^{2}\left(\hat{x}_{i}\right) \oplus \mathcal{S}^{2}\left(\hat{y}_{i}\right)$, где $\mathcal{S}^{2}\left(\hat{c}_{i}\right)$ - единичная сфера вектора $\vec{c}$ в $\mathcal{R}^{3}$. Такие гармоники мы запишем следующим образом:

$$
\mathcal{Y}_{\alpha}^{\varepsilon}\left(\hat{x}_{i}, \hat{y}_{i}\right) \equiv\left[Y_{l_{x_{i}}}^{m_{x_{i}}}\left(\hat{x}_{i}\right) \otimes Y_{l_{y_{i}}}^{m_{y_{i}}}\left(\hat{y}_{i}\right)\right]^{l, m}=(-1)^{l_{x_{i}}+l_{y_{i}}} \mathcal{Y}_{\alpha}^{\varepsilon}\left(-\hat{x}_{i},-\hat{y}_{i}\right)
$$

где компоненты мультииндекса $\alpha \equiv\left\{l_{x_{i}}, l_{y_{i}}\right\}$ подчинены неравенствам треугольника [18], в частности $\left|l_{x_{i}}-l_{y_{i}}\right| \leq l \leq l_{x_{i}}+l_{y_{i}}$, и дополнительному условию $l_{x_{i}}+l_{y_{i}}$ - четное (нечетное) число, если $\sigma=+1(-1)$. 
В качестве ортонормированного базиса пространства $\mathcal{A}^{\varepsilon}$ на единичной сфере $\mathcal{S}^{5}\left(\Omega_{i}\right)$ в $\mathcal{R}^{6}$ мы будем использовать полисферические гипергармоники [16] с квантовыми числами $\left\{L, l_{x_{i}}, l_{y_{i}}, l, m, \sigma\right\}$. Такие гипергармоники мы представим в виде произведений

$$
Y_{L, \alpha}^{\varepsilon}\left(\Omega_{i}\right) \equiv 2 \operatorname{cosec} 2 \varphi_{i} W_{L, \alpha}\left(\varphi_{i}\right) \mathcal{Y}_{\alpha}^{\varepsilon}\left(\hat{x}_{i}, \hat{y}_{i}\right)
$$

бисферических гармоник (8) и функций

$$
W_{L, \alpha}\left(\varphi_{i}\right) \equiv N_{L, \alpha}\left(\sin \varphi_{i}\right)^{l_{y_{i}}+1}\left(\cos \varphi_{i}\right)^{l_{x_{i}}+1} P_{n}^{\left(l_{y_{i}}+1 / 2, l_{x_{i}}+1 / 2\right)}\left(\cos 2 \varphi_{i}\right)
$$

Эти функции содержат полиномы Якоби $P_{n}^{(a, b)}$ и имеют единичную $\mathcal{L}_{[0, \pi / 2]}^{2}$-норму при соответствуюшем выборе множителя $N_{L, \alpha}$, что и подразумевается далее. По определению гипермомент $L$ является четным (нечетным) числом, если $\sigma=+1(-1)$, и принимает следующие значения: $L=l_{x_{i}}+l_{y_{i}}+2 n$, где $n=0,1, \ldots$

Следуюшие два свойства гипергармоник будут ключевыми для всех наших построений. Во-первых, гипергармоники являются собственными функциями [16]

$$
\mathbf{L}^{2} Y_{L, \alpha}^{\varepsilon}=L(L+4) Y_{L, \alpha}^{\varepsilon}
$$

оператора квадрата гипермомента $\mathbf{L}^{2}$, содержащегося в свободном гамильтониане

$$
H_{0}=-r^{5} \partial_{r}\left(r^{-5} \partial_{r}\right)+r^{-2} \mathbf{L}^{2}\left(\Omega_{i}\right)
$$

Во-вторых, гипергармоники $(9)$, записанные в разных $(i \neq k)$ представлениях $\left\langle\Omega_{i}\right|$ и $\left\langle\Omega_{k}\right|$, связаны унитарным преобразованием [19]

$$
\left\langle\Omega_{i} \mid Y_{\alpha, L}^{\varepsilon}\right\rangle \equiv Y_{L, \alpha}^{\varepsilon}\left(\Omega_{i}\right)=\sum_{\alpha^{\prime}}\left\langle\alpha \mid \alpha^{\prime}\right\rangle_{l, L} Y_{L, \alpha^{\prime}}^{\varepsilon}\left(\Omega_{k}\right)
$$

Здесь и далее подразумевается, что $i=1,2,3$, а индексы $\alpha \equiv\left\{l_{x_{i}}, l_{y_{i}}\right\}$ и $\alpha^{\prime} \equiv\left\{l_{x_{k}}, l_{y_{k}}\right\}$ могут принимать все значения, допустимые при заданных $l$ и $L$.

Преобразование (13) сохраняет квантовые числа $l, m, \sigma$ и $L$ и содержит коэффициенты Рейнала-Реваи [19]. Эти коэффициенты определены как интегралы перекрытия

$$
\begin{aligned}
\left\langle\alpha \mid \alpha^{\prime}\right\rangle_{l, L}\left(\gamma_{k, i}\right) & \equiv\left\langle l_{x_{i}}, l_{y_{i}} \mid l_{x_{k}}, l_{y_{k}}\right\rangle_{l, L}\left(\gamma_{k, i}\right) \equiv \\
& \equiv\left\langle Y_{L, \alpha}^{\varepsilon}\left(\Omega_{i}\right) \mid Y_{L, \alpha^{\prime}}^{\varepsilon}\left(\Omega_{k}\right)\right\rangle \equiv \int_{S^{5}} d \Omega_{i}\left(Y_{L, \alpha}^{\varepsilon}\left(\Omega_{i}\right)\right)^{*} Y_{L, \alpha^{\prime}}^{\varepsilon}\left(\Omega_{k}\right)
\end{aligned}
$$

и поэтому являются действительными функциями соответствующих кинематических углов (7).

\section{3. ЛОЖНЫЕ РЕШЕНИЯ}

Ложные решения подчинены условиям (4) и (5). Сначала исследуем условие (4). 
В теории Фаддеева каждая фаддеевская компонента $\Psi_{i}$ и уравнение, определяюшее ее в системе (1), записываются в соответствуюшем собственном координатном представлении $\left\langle r, \Omega_{i}\right|$. Поэтому в сумме $(4)$ каждая $(i=1,2,3)$ функция $S_{i}$ имеет аргументы $r$ и $\Omega_{i}$, а в каждом из уравнений (1) сумма этих функций записывается в соответствующем представлении $\left\langle r, \Omega_{i}\right|$. Следовательно, условие (4) означает, что такая сумма должна быть нулевой в каждом из трех $(i=1,2,3)$ наборов координат:

$$
\sum_{k=1}^{3}\left\langle r, \Omega_{i} \mid S_{k}\left(r, \Omega_{k}\right)\right\rangle=0
$$

Так как гиперрадиус $r$ является общей координатой для всех этих наборов и инвариантен относительно любых перестановок частиц, то квантовое число $L$, сопряженное c $r$, сохраняется при переходе от одного представления к другому. Это обстоятельство позволяет нам сушественно упростить задачу (15).

Сведем ее к счетной совокупности конечномерных линейных уравнений для гиперрадиальных компонент $S_{i, L, \alpha}$ разложений искомых решений по базису (9):

$$
\left\langle r, \Omega_{i} \mid S_{i}\right\rangle=r^{-2} \sum_{L} \sum_{\alpha} S_{i, L, \alpha}(r) Y_{L, \alpha}^{\varepsilon}\left(\Omega_{i}\right)
$$

Для этого подставим (16) в (15). Полученные уравнения спроецируем на базис (9). Далее учтем его ортонормированность, связи (13) и определения (14). Тогда для каждого значения $L$ получим линейную и однородную систему уравнений

$$
S_{i, L, \alpha}(r)+\sum_{k \neq i} \sum_{\alpha^{\prime}}\left\langle\alpha \mid \alpha^{\prime}\right\rangle_{l, L} S_{i, L, \alpha^{\prime}}(r)=0
$$

Здесь $i=1,2,3$, а $\alpha$ и $\alpha^{\prime}$ принимают все значения, допустимые при заданных $l, \sigma$ и $L$. Поэтому матрица $\mathbf{M}^{L}$ этой системы имеет конечную размерность. Она равна утроенному числу гипергармоник (9) с данными квантовыми числами $l$ и $L$. Из теории матрищ [21] известно, что система (17) имеет $N^{L}=\operatorname{dim} \mathbf{M}^{L}-\operatorname{rank} \mathbf{M}^{L}$ нетривиальных и линейно независимых решений тогда и только тогда, когда

$$
\operatorname{det} \mathbf{M}^{L}=0 .
$$

Пусть $\mathcal{B}$ - множество значений $L$, при которых выполняются условия (18). Если это множество пустое $(\mathcal{B}=\varnothing)$, т.е. $\operatorname{det} \mathbf{M}^{L} \neq 0$ при любом $L$, то не сушествует отличных от нуля функций (16), удовлетворяющих уравнениям (15), порожденных условием (4). В этом тривиальном случае уравнения (1) не имеют ложных решений в классе $\mathcal{A}^{\varepsilon}$.

Так как уравнения (15) сводятся к незацепляющимся системам уравнений (17), то их обшее решение (16) является линейной комбинацией

$$
S_{i}=\sum_{L \subset \mathcal{B}} C^{L} S_{i}^{L}\left(r, \Omega_{i}\right)
$$


некоторых числовых коэффишиентов $\mathrm{C}^{L}$ и частных решений

$$
S_{i}^{L}=\sum_{\alpha} S_{i, L, \alpha}(r) Y_{L, \alpha}^{\varepsilon}\left(\Omega_{i}\right)
$$

обладающих дополнительным к набору $\varepsilon$ квантовым числом $L$. Сформулируем доказанные результаты в виде леммы.

Лемма. В классе функиий $\mathcal{A}^{\varepsilon}$ система (15) имеет нетривиальные решения тогда и только тогда, когда $\mathcal{B} \neq \varnothing$. Все решения представимы в виде линейных комбинаций (19) функиий (20), гиперрадиальные компоненты которых подчинены условиям (17).

Теперь из всех решений (19) уравнений (4) выберем решения, удовлетворяюшие условию (5). Такие решения и будут ложными. В (5) подставим искомые решения $S_{i}$ в их обшем виде (19), (20). Используя (11) и (12), спроецируем получившиеся уравнения на базис (9). Введем новую переменную $z=\sqrt{E} r$. В итоге для каждой искомой гиперрадиальной компоненты $S_{i, L, \alpha}(z)$ с $L \subset \mathcal{B}$ получаем уравнение Бесселя [22] с индексом $\nu=L+2$. Оно имеет нетривиальные регулярные решения лишь при действительном $z>0$, т.е. при $E>0$. Все такие решения представимы в виде

$$
S_{i, L, \alpha}=D_{i, \alpha}^{L} J_{L+2}(\sqrt{E} r)
$$

где $J_{L+2}(\sqrt{E} r)$ - регулярная функция Бесселя, а $D_{i, \alpha}^{L}-$ некоторые числовые множители. При каждом заданном $L \subset \mathcal{B}$ функции (21) зависят от $r$ одинаковым образом, поэтому они удовлетворяют системе (17) тогда и только тогда, когда коэффициенты $D_{i, \alpha}^{L}$ подчиняются системе уравнений

$$
D_{i, \alpha}^{L}+\sum_{k \neq i} \sum_{\alpha^{\prime}}\left\langle\alpha \mid \alpha^{\prime}\right\rangle_{l, L} D_{k, \alpha^{\prime}}^{L}=0
$$

которая имеет ту же самую матрицу $\mathbf{M}^{L}$, что и система (17).

Подставив $(21)$ в $(20)$, получаем ложное решение с квантовыми числами $\varepsilon$ и $L \subset \mathcal{B}$

$$
S_{i}^{L}=r^{-2} J_{L+2}(\sqrt{E} r) \sum_{\alpha} D_{i, \alpha}^{L} Y_{L, \alpha}^{\varepsilon}\left(\Omega_{i}\right)
$$

Подставив все эти частные ложные решения в (19), получаем представление для обшего ложного решения

$$
S_{i}=r^{-2} \sum_{L \subset \mathcal{B}} C^{L} J_{L+2}(\sqrt{E} r) \sum_{\alpha} D_{i, \alpha}^{L} Y_{L, \alpha}^{\varepsilon}\left(\Omega_{i}\right)
$$

В силу леммы и доказанных вьше соотношений (21)-(24) имеет место следующий критерий сушествования ложных решений. 
Tеорема 1. В классе $\mathcal{A}^{\varepsilon}$ ложные решения $\left\{S_{1}, S_{2}, S_{3}\right\}$ уравнений (1) существуют тогда и только тогда, когда $E>0$, a $\mathcal{B} \neq \varnothing$. Все ложные решения представимы в виде сумм (24), содержащих произвольные коэффициенты $C^{L}$ и коэффициенты $D_{i, \alpha}^{L}$, подчиненнье условиям (21).

Теперь докажем, что все ложные решения обладают дополнительным к набору $\varepsilon$ квантовым числом. Пусть $\mathcal{S}^{\varepsilon}$ и $\mathcal{U}^{\varepsilon}$ - линейные подпространства пространства $\mathcal{A}^{\varepsilon}$, базисы которых на $S^{5}$ в $\mathcal{R}^{6}$ образуют гипергармоники $(9)$ с $L \subset \mathcal{B}$ и соответственно с $L \not \subset \mathcal{B}$. Тогда операторы

$$
P_{s} \equiv \sum_{L \subset \mathcal{B}} \sum_{\alpha}\left|Y_{L, \alpha}^{\varepsilon}\left(\Omega_{i}\right)\right\rangle\left\langle Y_{L, \alpha}^{\varepsilon}\left(\Omega_{i}\right)\left|, \quad P_{u} \equiv \sum_{L \not \subset \mathcal{B}} \sum_{\alpha}\right| Y_{L, \alpha}^{\varepsilon}\left(\Omega_{i}\right)\right\rangle\left\langle Y_{L, \alpha}^{\varepsilon}\left(\Omega_{i}\right)\right|
$$

являются проекторами на $\mathcal{S}^{\varepsilon}$ и соответственно на $\mathcal{U}^{\varepsilon}$. Поэтому пространство $\mathcal{S}^{\varepsilon}$ ортогонально пространству $\mathcal{U}^{\varepsilon}$ относительно интегрирования на сфере $S^{5}$ по совокупности гиперуглов $\Omega_{i}$.

Так как преобразования (13) унитарны, то представления (25) проекторов $P_{s}$ и $P_{u}$ инвариантны относительно индекса $i$. Учтя это, подействуем проекторами на ложные решения (24). В результате получим

$$
P_{s} S_{i}=p_{s} S_{i}, \quad p_{s}=1, \quad P_{u} S_{i}=0 .
$$

Значит, компоненты $S_{i}$ всех ложных решений $\left\{S_{1}, S_{2}, S_{3}\right\}$ являются собственными функциями оператора $P_{s}$, отвечаюшими одному и тому же собственному числу $p_{s}=1$ и принадлежашими множеству $\mathcal{S}^{\varepsilon}$. Следовательно, в подпространстве $\mathcal{U}^{\varepsilon}$ уравнения $\Phi$ аддеева (1) не могут иметь ложных решений. Используя этот факт, выведем из уравнений Фаддеева модифицированные уравнения, не имеющие ложных решений.

Так как $\mathcal{U}^{\varepsilon}$ является ортогональным дополнением $\mathcal{S}^{\varepsilon}$ до $\mathcal{A}^{\varepsilon}$, то компоненты любого нетривиального решения уравнений (1) представимы в виде сумм их проекций на эти подпространства:

$$
\Psi_{i}=P_{u} \Psi_{i}+P_{s} \Psi_{i}
$$

Используя это представление, докажем следуюшие соотношения:

$$
\sum_{k=1}^{3} P_{u} \Psi_{k} \neq 0, \quad \sum_{k=1}^{3} P_{s} \Psi_{k}=0 .
$$

Предположим противное: пусть при любом $i=1,2,3$ и некоторых ненулевых функциях $R_{i}$ из $\mathcal{S}^{\varepsilon}$ верны следуюшие соотношения:

$$
\begin{aligned}
& \sum_{k=1}^{3}\left\langle r, \Omega_{i} \mid P_{u} \Psi_{k}\left(r, \Omega_{k}\right)\right\rangle=0, \\
& \sum_{k=1}^{3}\left\langle r, \Omega_{i} \mid P_{s} \Psi_{k}\left(r, \Omega_{k}\right)\right\rangle=R_{i} .
\end{aligned}
$$


Подставим в них $\Psi_{i}$ и $R_{i}$, разложенные по базису (9), а $P_{s}$ и $P_{u}$ представим в виде (25). Используя (13) и (14), спроецируем полученные соотношения на базис (9). В результате исходные равенства (29) сведутся к совокупности уравнений, содержащих гиперрадиальные компоненты $\Psi_{i, L, \alpha}$ и $R_{i, L, \alpha}$ функций $\Psi_{i}$ и $R_{i}$. Для каждого фиксированного $L$ имеем

$$
\Psi_{i, L, \alpha}+\sum_{k \neq i} \sum_{\alpha^{\prime}}\left\langle\alpha \mid \alpha^{\prime}\right\rangle_{l, L} \Psi_{k, L, \alpha^{\prime}}= \begin{cases}0, & L \not \subset \mathcal{B}, \\ R_{i, L, \alpha}, & L \subset \mathcal{B} .\end{cases}
$$

Если $L \not \subset \mathcal{B}$, то однородная система (30) имеет лишь тривиальное решение, т.к. ее матрица $M^{L}$ не вырождена. В случае $L \subset \mathcal{B}$ неоднородная система (30) имеет вырожденную матрицу $M^{L}$ и несовместна по альтернативе Фредгольма [21]. Полученные противоречия доказывают справедливость соотношений (28).

Введем обозначение $U_{i} \equiv P_{u} \Psi_{i}$. Используя (25)-(28), спроецируем уравнения (1) на подпространство $\mathcal{U}^{\varepsilon}$. Тогда получим модифицированные уравнения

$$
\left(H_{0}-E\right) U_{i}=-P_{u} V_{i} \Psi=-P_{u} V_{i} \sum_{k=1}^{3} U_{k}
$$

Все решения этих уравнений принадлежат пространству $\mathcal{U}^{\varepsilon}$. Соответствуюшая волновая функция $\Psi=U_{1}+U_{2}+U_{3}$ всегда отлична от нуля.

Просуммируем доказанные утверждения в виде теоремы.

Теорема 2. В классе $\mathcal{A}^{\varepsilon}$ фаддеевские компоненты $\Psi_{i}$ представимы в виде сумм (27) ортогональных слагаемых $P_{u} \Psi_{i}$ u $P_{s} \Psi_{i}$, которые удовлетворяют соотношениям (28). Слагаемые $U_{i} \equiv P_{u} \Psi_{i}$ подчинены системе уравнений (31), которая не имеет ложних решений.

\section{4. ПРИМЕРЫ ЛОЖНЫХ РЕШЕНИЙ}

4.1. Центральные потенциалы. Заметим, что только при $l, L=0$ или $l=1$, $L=2$ индекс $\alpha$ гипергармоник (9) может принимать лишь одно значение $\alpha=\{0,0\}$ или соответственно $\alpha=\{1,1\}$. В этих случаях имеется только один коэффициент Рейнала-Реваи отличный от нуля: $\langle\alpha \mid \alpha\rangle_{l, L}\left(\gamma_{k, i}\right)=1$. Поэтому связи (13) вырождаются в равенства

$$
Y_{L, \alpha}^{\varepsilon}\left(\Omega_{i}\right)=Y_{L, \alpha}^{\varepsilon}\left(\Omega_{k}\right)
$$

где $l, L=0$ или $l=1, L=2$, а все элементы матриц $\mathbf{M}^{0}$ и $\mathbf{M}^{2}$ систем (21) равны единице. Следовательно, при $L=0,2$ имеем $\operatorname{dim} \mathbf{M}^{L}=3, \operatorname{rank} \mathbf{M}^{L}=1$. В обоих случаях обшее решение систем (21) таково: $D_{2, \alpha}^{L}$ и $D_{3, \alpha}^{L}$ - произвольные числа, не равные нулю одновременно, а $D_{1, \alpha}^{L}=-D_{2, \alpha}^{L}-D_{3, \alpha}^{L}$. По формуле (23) находим фаддеевские компоненты

$$
S_{i}^{L}=r^{-2} J_{L+2}(\sqrt{E} r)\left(D_{2, \alpha}^{L}\left(\delta_{i, 2}-\delta_{i, 1}\right)+D_{3, \alpha}^{L}\left(\delta_{i, 3}-\delta_{i, 1}\right)\right) Y_{L, \alpha}^{\varepsilon}\left(\Omega_{i}\right)
$$

частных ложных решений, имеющих квантовые числа $l, m=0, \sigma=1, L=0$ и $\alpha=\{0,0\}$ или $l=1, m=0, \pm 1, \sigma=1, L=2$ и $\alpha=\{1,1\}$. 
Найденные решения (33) не зависят от масс частиц и вырождаются в тривиальные, если все три частицы тождественные. Последнее утверждение стоит доказать.

Для состояния $\varepsilon$ системы трех тождественных бозонов (фермионов) компоненты физического решения уравнений Фаддеева (1), а значит, и ложного решения, подчинены дополнительным условиям [2], обеспечиваюшим полную (анти)симметрию волновой функции относительно перестановок частиц в любой паре. Запишем эти условия для компонент ложного решения:

$$
S_{1}\left(\vec{x}_{1}, \vec{y}_{1}\right)= \pm S_{1}\left(-\vec{x}_{1}, \vec{y}_{1}\right), \quad S_{i}\left(\vec{x}_{i}, y_{i}\right)=S_{1}\left(\vec{x}_{i}, \vec{y}_{i}\right), \quad i=2,3
$$

Они означают, что в случае (фермионов) бозонов первая фаддеевская компонента должна быть (анти)симметрична относительно перестановки частищ с номерами 2 и 3 , и в обоих случаях вторая и третья компоненты должны быть равны первой, записанной в собственных для этих компонент наборах координат.

Подставим (33) в (34) и учтем (32). Получим, что из всех ложных решений (33) только тривиальное $\left(D_{i, \alpha}^{L}=0, i=1,2,3\right)$ удовлетворяет условиям $(34)$.

Используя базисы (9) и (10), представим потенциалы в пространстве $\mathcal{A}^{\varepsilon}$ операторными рядами

$$
V_{i}=\sum_{\lambda_{i}=0}^{\infty} V^{\lambda_{i}}\left(\vec{x}_{i}\right),
$$

в которых по определению

$$
\begin{aligned}
V_{i}^{\lambda_{i}}\left(\vec{x}_{i}\right) & \equiv \sum_{l_{y_{i}}} \sum_{L, L^{\prime}}\left|Y_{L, \alpha}^{\varepsilon}\left(\Omega_{i}\right)\right\rangle V_{i, \alpha}^{L, L^{\prime}}(r)\left\langle Y_{L^{\prime}, \alpha}^{\varepsilon}\left(\Omega_{i}\right)\right|, \\
V_{i, \alpha}^{L, L^{\prime}}(r) & \equiv \int_{0}^{\pi / 2} d \varphi_{i} W_{L, \alpha}\left(\varphi_{i}\right) V_{i}\left(r \cos \varphi_{i}\right) W_{L^{\prime}, \alpha}\left(\varphi_{i}\right), \quad \alpha=\left\{\lambda_{i}, l_{y_{i}}\right\} .
\end{aligned}
$$

Операторы (36) обладают следуюшими проекционными свойствами:

$$
\left\langle\Omega_{i}\left|V_{i}^{\lambda_{i}}\right| Y_{L^{\prime}, \alpha}^{\varepsilon}\left(\Omega_{i}\right)\right\rangle=\delta_{\lambda_{i}, l_{x_{i}}} \sum_{L} V_{i, \alpha}^{L, L^{\prime}} Y_{L, \alpha}^{\varepsilon}\left(\Omega_{i}\right), \quad \alpha=\left\{l_{x_{i}}, l_{y_{i}}\right\}
$$

Следовательно, они сохраняют орбитальный момент $\lambda_{i}$ пары частиц с номером $i$. Такие потенциалы широко применяются в ядерной физике низких энергий [17]. При $\lambda_{i}=0,1,2$ они называются соответственно $S$-, $P$ - и $D$-волновыми потенциалами. Формулы (35)-(37) показывают, как перейти от сферически-симметричных потенциалов к $\lambda$-волновым. Для этого нужно запретить индексам $\alpha$ и $\alpha^{\prime}$ принимать где-либо значения, отличные от $\left\{\lambda_{i}, l_{y_{i}}\right\}$ и $\left\{\lambda_{i}, l_{y_{k}}\right\}$.

4.2. $S$-волновые потенциалы. В случае $S$-волновых потенциалов, кроме набора $\varepsilon$, сохраняются квантовые числа $l_{x_{i}}=0$ и $l_{y_{i}}=l$. При любых $l$ и $L$ индексы $\alpha$ и $\alpha^{\prime}$ в формуле (13) преобразования гипергармоник, в системе (21) для коэффициентов $D_{i, \alpha}^{L}$ ложных решений и в общих представлениях (24) этих решений могут принимать 
лишь одно значение $\alpha=\alpha^{\prime}=\{0, l\}$. Поэтому матрица $\mathbf{M}^{L}$ этой системы всегда имеет размерность, равную трем. Все ее недиагональные элементы находим по известной формуле [20]

$$
\begin{aligned}
M_{k, i}^{L} & \equiv\langle 0, l \mid 0, l\rangle_{l, L}\left(\gamma_{k, i}\right)= \\
& =\left(-\cos \gamma_{k, i}\right)^{l} P_{(L-l) / 2}^{(l+1 / 2,1 / 2)}\left(-\cos 2 \gamma_{k, i}\right) / P_{(L-l) / 2}^{(l+1 / 2,1 / 2)}(-1) .
\end{aligned}
$$

Условие (18) сводим к равенствам

$$
\operatorname{det} M^{L}=1-\left(M_{2,1}^{L}\right)^{2}-\left(M_{1,3}^{L}\right)^{2}-\left(M_{3,2}^{L}\right)^{2}+2 M_{2,1}^{L} M_{1,3}^{L} M_{3,2}^{L}=0 .
$$

Пусть $l=0, L=2$. Тогда $\alpha=\alpha^{\prime}=\{0,0\}$. С помошью (38) и (39) находим, что при любых массах частищ $M_{k, i}^{2}=\cos 2 \gamma_{k, i}, \operatorname{rank} \mathbf{M}^{2}=2, \operatorname{adet} \mathbf{M}^{2}=0$. Далее используем два свойства $\gamma_{2,1}, \gamma_{1,3}, \gamma_{3,2}>0$ и $\gamma_{3,2}=\pi-\gamma_{2,1}-\gamma_{1,3}$ кинематических углов (7) и показываем, что все решения системы (22) пропорциональны решению

$$
D_{i, \alpha}^{L}=\sin \left(L\left(\gamma_{2,1}+\gamma_{1,3}\right) \delta_{i, 1}-(-1)^{L} L\left(\gamma_{1,3} \delta_{i, 2}+\gamma_{2,1} \delta_{i, 3}\right)\right) .
$$

Подставив его в (23), получаем соответствуюшее частное ложное решение

$$
\begin{aligned}
S_{i}^{L}= & r^{-2} J_{L+2}(\sqrt{E} r) Y_{L, \alpha}^{\varepsilon}\left(\Omega_{i}\right) \times \\
& \times \sin \left(L\left(\gamma_{2,1}+\gamma_{1,3}\right) \delta_{i, 1}-(-1)^{L} L\left(\gamma_{1,3} \delta_{i, 2}+\gamma_{2,1} \delta_{i, 3}\right)\right) .
\end{aligned}
$$

Оно имеет квантовые числа $l, l_{x_{i}}, l_{y_{i}}=0, \sigma=1$ и $L=2$ и зависит от масс частиц посредством кинематических углов.

Пусть $l, L=1$. Тогда всюду $\alpha=\alpha^{\prime}=\{0,1\}$. В силу (38) и (39) имеем $M_{k, i}^{1}=-\cos \gamma_{k, i}, \operatorname{rank} \mathbf{M}^{1}=2, a \operatorname{det} \mathbf{M}^{1}=0$. Находим решение системы (21) и соответствуюшее ему частное ложное решение (23). Записываем их формулами (40) и (41), в которых теперь $l_{y_{i}}, l, L=1, \sigma=-1$ и $\alpha=\{0,1\}$.

Пусть $l$ и $L$ любые, но частицы имеют одинаковые массы. Тогда $\alpha=\alpha^{\prime}=\{0, l\}$ и согласно (7) $\left|\gamma_{k, i}\right|=\pi / 3$ для любых $k$ и $i$. Поэтому все недиагональные элементы (38) матрицы $M^{L}$ равны друг другу и условие (39) упрошается: при каждом фиксированном $l$ оно сводится к диофантову уравнению

$$
(-2)^{l-1} P_{(L-l) / 2}^{(l+1 / 2,1 / 2)}(-1)=P_{(L-l) / 2}^{(l+1 / 2,1 / 2)}(1 / 2)
$$

относительно $L$. При $l=0$ или $l=1$ имеется по одному решению: $L=2$ или $L=1$, а соответствуюшие частные ложные решения (41) принимают вид

$$
S_{i}^{L}=(-1)^{L+1}(\sqrt{3} / 2) r^{-2} J_{L+2}(\sqrt{E} r) Y_{L, \alpha}^{\varepsilon}\left(\Omega_{i}\right) .
$$

Эти решения удовлетворяют условиям (34), обеспечиваюшим симметрию волновой функции состояния $\varepsilon$ трех тож дественных бозонов, и воспроизводят известные ложные решения $[11,12,14]$.

Как известно [2], в случае $S$-волновых потенциалов пространственная часть волновой функции квартетного по спину $(s=3 / 2)$ рассеяния нейтрона на дейтроне в состоянии с $l=0$ полностью симметрична. Фаддеевские компоненты этой части волновой функции подчинены системе (1). Такая система имеет ложное решение (43) с $L=2$. 
4.3. $P$-волновые потенциалы. Пусть потенциалы $P$ волновые, а массы частиц произвольны. Теперь, кроме чисел $l$ и $m$, сохраняется $l_{x_{i}}=1$, a $l_{y_{i}}=l, l \pm 1$ и, вообще говоря, не сохраняется. Следовательно, индексы $\alpha$ и $\alpha^{\prime}$ в формуле (13) преобразования гипергармоник, в системе (21) для коэффишиентов $D_{i, \alpha}^{L}$ ложных решений и в обших представлениях (24) этих решений могут принимать не более трех значений: $\alpha, \alpha^{\prime}=\{1, l\}$, $\{1, l \pm 1\}$. Поэтому размерность матрицы $\mathbf{M}^{L}$ этой системы не может превышать девять. Исключение составляют исследованный выше случай $l=1$ и $L=2$ и случаи $l=0, L=2$ и $l, L=1$. Рассмотрим их.

Пусть $l=0, L=2$. Тогда $\alpha=\alpha^{\prime}=\{1,1\}$. По формулам, приведенным в [16], находим все недиагональные элементы матрицы $\mathbf{M}^{2}$ системы (21). Получаем $M_{i, i}^{2}=1$ и $M_{k, i}^{2}=\cos 2 \gamma_{k, i}$. Эта матрица совпадает с матрицей $\mathbf{M}^{2}$, исследованной ранее в случае $S$-волновых потенциалов. Поэтому формулы (40) и (41) верны и в рассматриваемом случае, когда $l=0, l_{x_{i}}, l_{y_{i}}=1$ и $L=2$.

Пусть $l, L=1$. Тогда $\alpha=\alpha^{\prime}=\{1,0\}$. Теперь матрица $\mathbf{M}^{1}$ совпадает с матрицей $\mathbf{M}^{1}$, рассмотренной выше в случае $S$-волновых потенциалов. Значит, формулы (40) и (41) справедливы и в рассматриваемом случае, т.е. при $l_{x_{i}}, l, L=1$ и $l_{y_{i}}=0$.

В обоих рассмотренных случаях $(l=0, L=2$ и $l, L=1)$ частные ложные решения (41) при равных массах частиш сводятся к соответствуюшим ложным решениям (43). Последние удовлетворяют условиям (34), обеспечивающим полную антисимметрию волновой функции трех тождественных фермионов.

Рассмотрим систему из трех нейтронов в состоянии с $l=1, s=3 / 2$ и полным моментом $(\vec{j}=\vec{l}+\vec{s})$, равным $5 / 2$. В таком состоянии нейтроны взаимодействуют только посредством $P$-волновых потенциалов, а пространственная часть их волновой функции полностью антисимметрична. Ее фаддеевские компоненты подчинены системе (1), которая имеет ложное решение (43) с $L=1$.

\section{5. ЗАКЛЮЧЕНИЕ}

Основные результаты настояшей работы были сформулированы в виде леммы и теорем 1,2 . Сравним эти результаты с известными.

Мы вывели необходимые и достаточные условия $(E>0$ и $\mathcal{B} \neq \varnothing)$ сушествования ложных решений. Первое условие $(E>0)$ давно известно [6].

Мы доказали, что фаддеевские компоненты являются суммами (27) двух ортогональных слагаемых. Эти слагаемые принадлежат разным подпространствам $\mathcal{S}^{\varepsilon}$ и $\mathcal{U}^{\varepsilon}$ и удовлетворяют соотношениям (28). Такое строение фаддеевских компонент было доказано в [9] другим более сложным способом. Согласно терминологии, принятой в [9], найденные нами пространства $\mathcal{U}^{\varepsilon}$ и $\mathcal{S}^{\varepsilon}$ можно назвать пространством физических и ложных решений уравнений Фаддеева. Мы показали, что эти уравнения в физическом пространстве имеют вид (31).

Мы предложили простой способ построения ложных решений в явном виде для широкого класса трехчастичных систем. Демонстрируя этот способ, мы нашли новые частные ложные решения (33) и (41) и воспроизвели известные ложные решения.

В заключение стоит отметить следуюшее.

В основе нашего подхода лежит достаточно развитьй метод гипергармоник. Поэтому наш подход нетрудно обобшить на более реалистический случай, когда частицы 
обладают и дискретными степенями свободы, такими как спин и изоспин. Для такого обобщения необходимо использовать соответствующие гипергармоники, описывающие и эти степени свободы. Техника построения таких гипергармоник достаточно развита как для трех-, так и для четырехчастичных систем [16, 17]. Поэтому предложенный нами подход также нетрудно обобщить для исследования ложных решений задачи четырех частиц. Одно из таких решений было недавно найдено в [15].

Автор глубоко признателен М. Декеру и А.К. Мотовилову за ценные замечания.

\section{Список литературы}

[1] Л.Д. Фаддеев. Математические вопросы квантовой теории рассеяния для системы трех частиц // Труды МИАН.. М.: Наука, 1963. Т. 69.

[2] С.П.Меркурьев, Л.Д.Фаддеев. Квантовая теория рассеяния для систем нескольких частиц. М.: Наука, 1985.

[3] S.P.Merkuriev // Ann. Phys. 1980. V. 130. № 2. P. 395-426.

[4] А.А.Квицинский, Ю.А.Куперин, С.П.Меркурьев и др. // ЭЧАЯ. 1986. Т. 17. № 2. C. $267-317$.

[5] А.А.Квичинский, В.В.Кострыкин, С.П.Меркурьев // ЭЧАЯ. 1990. Т. 21. №6. C. $1301-1359$.

[6] S.K.Adhikari, W.Glöckle // Phys. Rev. 1979. V. C19. №3. P. 616-630.

[7] F.S.Levin // Ann. of Phys. 1980. V. 130. № 1. P. 139-163.

[8] J.W.Evans // J. Math. Phys. 1981. V. 22. № 8. P. 1672-1686.

[9] J.W.Evans, D.K.Hoffman // J. Math. Phys. 1981. V. 22. № 12. P. 2858-2871.

[10] S.L.Yakovlev. Some spectral properties for the Faddeev matrix operator and its adjoint one: Preprint LTPE Orsay 91/14, 1991.

[11] J.F.Friar, B.F.Gibson, G.L.Payne // Phys. Rev. 1980. V. C22. №1. P. 284-286.

[12] В.В.Пупышев // ТМФ. 1989. Т. 81. № 1. С. 86-93.

[13] V.V.Pupyshev // Phys. Lett. 1989. V. A140. № 4. P. 151-154.

[14] V.V.Pupyshev // Few Body System. 1990. V. 8. № 2. P. 105-122.

[15] M. Fabre de la Ripelle, S. Y.Larsen // Few Body System. 1992. V. 13. № 3. P. 199-206.

[16] Р.И.Джибути, Н.Б.Крупенникова. Метод гиперсферических функций в квантовой механике нескольких тел. Тбилиси: Мецниереба, 1984.

[17] Р.И.Джибути, К.В.Шитикова. Метод гиперсферических функций в атомной и ядерной физике. М.: Энергоатомиздат, 1993.

[18] L.C.Biedenharn, J.D.Louck. Angular momentum in quantum physics. V. 1. Massachusetts: Addison-Wesley Publishing Company, 1981.

[19] J.Raynal, J.Revai // Nuovo Cim. 1970. V. A68. №4. P. 612-622.

[20] В.Д.Эфрос // ЯФ. 1973. Т. 17. №1. С. 210-224.

[21] P.Lankaster. Theory of matrices. New York-London: Academic Press, 1969.

[22] Г.Бейтмен, А.Эрдейи. Высшие трансцендентные функции. Т. 1. М.: Наука, 1974.

Объединенный институт ядерных исследований

Поступила в редакцию 19.VI.1995 г

\section{V.V.Pupyshev \\ SPURIOUS SOLUTIONS OF FADDEEV EQUATIONS WITH CENTRAL POTENTIALS}

Spurious solutions of the differential Faddeev equations for the system of three different particles interacting via central potentials are investigated within the hyperharmonics approach. The criterion for existence of the spurious solutions is proven and the method for the construction of these solutions is proposed. The results obtained are exemplified. 